\title{
Editorial - ESA 2016 Special Issue
}

This special issue contains four among the top papers that were presented (in a preliminary form) at the 24th European Symposium on Algorithms (ESA), Engineering and Applications Track (Track B), that took place on 22-24 August 2016 in Aarhus (Denmark). ESA is a premier conference on algorithms, covering research in the broad spectrum of design, analysis, engineering, and applications of algorithms and data structures.

The articles in this special issue were invited based on their evaluation by the ESA 2016 Track B program committee, as well as on extensive discussions among the members of the same committee. All articles are widely expanded versions of their preliminary counterparts presented at ESA 2016, are indicative of the quality of ESA 2016 papers, and went through the standard rigorous refereeing process of the ACM Journal of Experimental Algorithmics before being accepted to this special issue.

The contents of the four articles comprising the special issue of the ACM fournal of Experimental Algorithmics on ESA 2016 Track B are summarized as follows:

- In the article "KADABRA is an ADaptive Algorithm for Betweenness via Random Approximation," Michele Borassi and Emanuele Natale present a new algorithm (KADABRA) to approximate the betweenness centrality in directed and undirected graphs. The new method outperforms all previous approaches on real-world complex networks. The efficiency of the new algorithm relies on two new theoretical contributions: (i) sampling shortest paths, based on a balanced bidirectional BFS, and (ii) a rigorous application of the adaptive sampling technique, which decreases the total number of shortest paths that need to be sampled to compute all betweenness centralities with a given absolute error.

- In the article "Better External Memory LCP Array Construction," Juha Kärkkäinen and Dominik Kempa present two new algorithms for computing the LCP array (that stores the lengths of the longest common prefixes between lexicographically adjacent suffixes) from the suffix array (a lexicographically sorted list of the suffixes of a text) in external memory. The augmentation of a suffix array with LCP constitutes a powerful tool in modern string processing. The authors demonstrate, through an experimental study, that the new algorithms are about a factor of two faster than the fastest previous one. In addition, they further engineer and improve the two new algorithms in several ways (speeding them up further using parallelism, reducing the disk usage by making them in-place, and modifying them to support large alphabets).

- In the article "BlockQuicksort: How Branch Mispredictions Don't Affect Quicksort," Stefan Edelkamp and Armin Weiss present a novel approach to address the problem of branch mispredictions (misses) in the quicksort algorithm. Their approach is based on the partial decoupling of control from data flow. In particular, their partition procedure incurs few branch mispredictions by storing results of comparisons in constant-size buffers. An accompanying experimental study shows that, on almost all random and non-random input data they considered, the new algorithm has a significant speedup compared to the standard gcc implementation of quicksort. On random input permutations, the new

(C) 2019 Copyright held by the owner/author(s).

1084-6654/2019/01-ART1.1

https://doi.org/10.1145/3298788 
algorithm is even faster than an implementation of the highly tuned Super Scalar Sample Sort, which uses a linear amount of additional space.

- In the article "SimBa: An Efficient Tool for Approximating Rips-Filtration Persistence via Simplicial Batch-Collapse," Tamal Dey, Dayu Shi, and Yusu Wang investigate the advantages and limitations of existing approaches for computing the persistence diagram or barcodes of a sequence of (so-called) Rips complexes that are used in topological analysis of big and complex data. Existing approaches do not scale well, especially in high dimensional data, as the size of the Rips complexes may become prohibitively large. Based on insights gained from the experiments, the authors propose a new algorithm, called SimBa, for approximating the persistent homology of Rips filtrations with quality guarantees. The new algorithm leverages a batch collapse strategy as well as a new sparse Rips-like filtration. An accompanying experimental study shows that, on a variety of low- and high-dimensional datasets, the new algorithm for approximating Rips filtration persistence is an order of magnitude faster than existing methods in practice, and the overall strategy offers a significant reduction in the size of the filtration.

I would like to thank the authors for submitting their articles to this special issue, as well as the reviewers for their excellent and timely work that improved the quality of the submissions. I would also like to thank Gonzalo Navarro (editor-in-chief of the ACM Journal of Experimental Algorithmics), Laura Lander (ACM Journals Manager) and Arriane Bustillo (from the editorial support services) for their constant support and the opportunity to publish this special issue. I hope that the readers will find the articles interesting and enjoyable.

Christos Zaroliagis

CTI \& University of Patras

Guest Editor 